Dans ce cas, la formule (12) nous donne:

$$
D=\sqrt[7]{\frac{0,046 \times 200 \times \overline{1,4^{3} \times 6000}}{560 \times 0.1 \times 450}}=\sqrt[7]{3,6}=1 \mathrm{~m} 20
$$

On trouve alors, avec la formule de Levy:

$$
J=0,{ }^{m 0018} \quad h=0,0018 \times 2000=3,{ }^{m 60}
$$

La vitesse de l'eau est ici de 1 m9).

Le pourcentage de la perte de charge brute est done:

$$
\frac{3,60}{560}=0,6 \text { pour } 100
$$

L. PIERRE,

Ingenieur I.E.G.

\section{INSTALLATIONS HYDR0-ELLETRIOUES}

\section{LAS INSTALLATIONS DE LA SOCHEIE ANONYLIE DES FORCES MOTRICLS DL BRUSI0 \\ (Suite)}

\section{Station de transformation de Fiattamala}

La ralléc du Poschiarino, parliculièrement étroite depuis Campocologno, lassail pou de choix pour la construclion d'un batiment d'une certane importance, il fallut cependant faire santer plusiens centaines de melres cubes de roche: pour pouvoir dégager l'emplacement nécessaime.

Ia station de Piallamala esl destinte álever, de 7.000 in 5o.000 vells, la lension du courant vemant de lusine de Brusio.

Flle se compose d"un rasle bitiment de 65 mètres de longueur stur ? I nètres de largeur, et d'une lourelle amnese

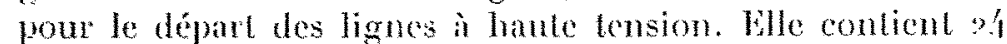
trankformateurs monophasés, prévus pour une puisance de 1.350 K. V. A., qui ont été foumis par la Société Nlioth ainsi dailleurs que lout l'upparcillage de la station.

CHOMBne bes Mesches. - Le tumnel contenant la double canalisalion triphasé dont it a été parlé précédemmont, aboulii an rez-de-chansséc de la shation d'où les conduiles d'arvive montent dans la chambere des mesures. Des conteaux permetlont de entper la camalisation on de remise ('u partlobe zes deux branclas. Ia stalion pout donc fonctionner arec les deux moitiós séparées an montées on paralleb. On na pas employé dinterupleus antonatiques, car lo déclenchement subit de la charge totale anail pu étre dangerenx pour linstalabion hydrauligue.

la chambre des mesures comporle, immédiatenent apres les conleaux de sectionmement, denx lableaux, munis chacun : de trois amperemères arec transformateurs d'intonsile, dont un pour chaque phase; - d'm volmètre ave commulateur ; - d'un rollmotre entegistremp - et de deux Wallmefres enregistreurs nontés en série el fournis par denx consleucteurs différents, rela alin d'avoir un double controble

la mène salle contiont igalement les ampètenètres el voltmètres des lignes de dépirl à 50.000 volts, ce qui permel do surveiller d'un seul point le fonctionnement ar lensemble.

les barres a 7 .ooo wolls sortaul de la chambre des mesumer, se rendenl dans la salle des transformateurs, qui sont nontés en ctoile par geompes de trois.

Chaque groupe de transformaleurs est pourvu d'm interrupleur tripolaire du colé 7 .ooo volts, et d'un interrupleur simple sur chaque phase de la haute tension. Tinterrupleur a 7.000 volls ef les trois intervupteurs à 5.000 volts de cha- que groupe peurent décheher automatiquenent an moyen diun disjoncteur à relai monté sur la basse tension, ce qui assure l'isolement compled d'un groupe en ras d'accident, el cmpeche les antres transformateurs de débiter sur ceux qui sont arariés.

Toules les camalisalions dedediques sonl séparés les unes des aulres par des cloisons en béton, c'est d'ailleurs la une règle absolue que l'on a suivie dans loutes les stalions cit sous-stations.

Traxsornategrs. - les transformateurs à noyaux velticaux (fig. 19 et 20$)$ sont à bain d'huile refroidie par circulalion d'ent. Au lieu du sepentin généralement adoplé, la sociéte Alioth entoure la parlie supérieure de la cave à hule d'une chemise en tole, dans laquelle l'ean froide, enlrant par la partic inférieure, et sortanl pas en hant, se lenouvelle constamment. Des nervures imtéricures permellem diacliver Io refroidissement. Avec ce systeme, on n'a pas à craindre les fuiles.
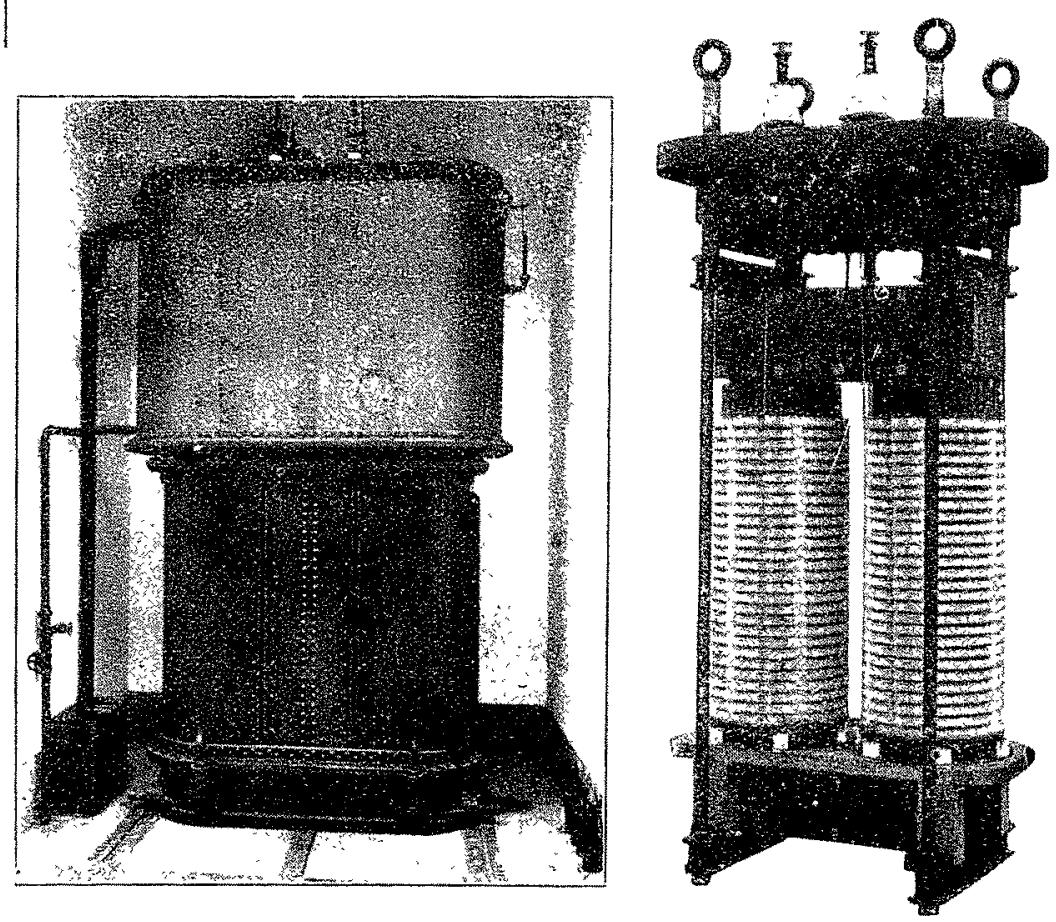

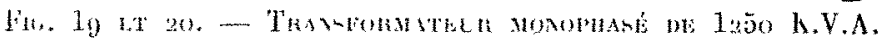

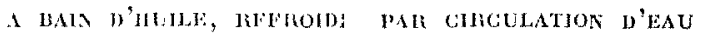

Iat juissance nommle des limisformaleurs est de r.250 K. V. A. Le rapport de trausformation de chaque groupe monté en éloile est, suivant la tension des aternateurs de la centralc,

$$
\frac{7500}{47000} \text { on } \frac{7700}{18300}
$$

Lemonlement hanle lansion exléricur est conslilué par 56 bobines empilécs, el sćpartes les unes des aulres par des cales isolantes en mécanite. De celle façon, la tension limito de 1.000 volls entre les extrémités d'uno même bobine, que Con simpose gónéralemont, n'est pas alteinle; de plus, les réparations sont grandentent facilitées, el le remplacomenl d'une bobine brûléc est une opération relalivement rapide.

les rendements imposés par lo eahier des charges, soit $97,5 \%$ a pleine clarge, el $96,5 \%$ a demi-charge, ont été facilement alteints ( $($ iq, 21).

les chutes de tension provues, at déteminces au moyen du diagrammo classique de Kapp,

$$
\begin{aligned}
\text { soit } \mathrm{x} \% \text { pour } \cos \% & =\overline{\mathrm{r}} \\
\text { ct } 2,2 \% \text { pour } \cos \% & =0,80 .
\end{aligned}
$$

n'onl pas élé dépasscés. 
L'élévation de température, mesurée par la méthode du thermomètre, n'a pas non plus dépassé les limites imposées, savoir:

$45^{\circ} \mathrm{C}$. ant-dessus de la tempéralure ambiante, en ulilisanl an litres d'eau par minute;

$6 n^{\circ} \mathrm{C}$. avec la mème quantilé d'eau, et une surcharge de $35 \%$ pendant deux houres.

$45^{\circ} \mathrm{C}$. avec 40 litres d'eau, of une surcharge de $25 \% \mathrm{p}^{\circ \mathrm{n}-}$ dant six heures.

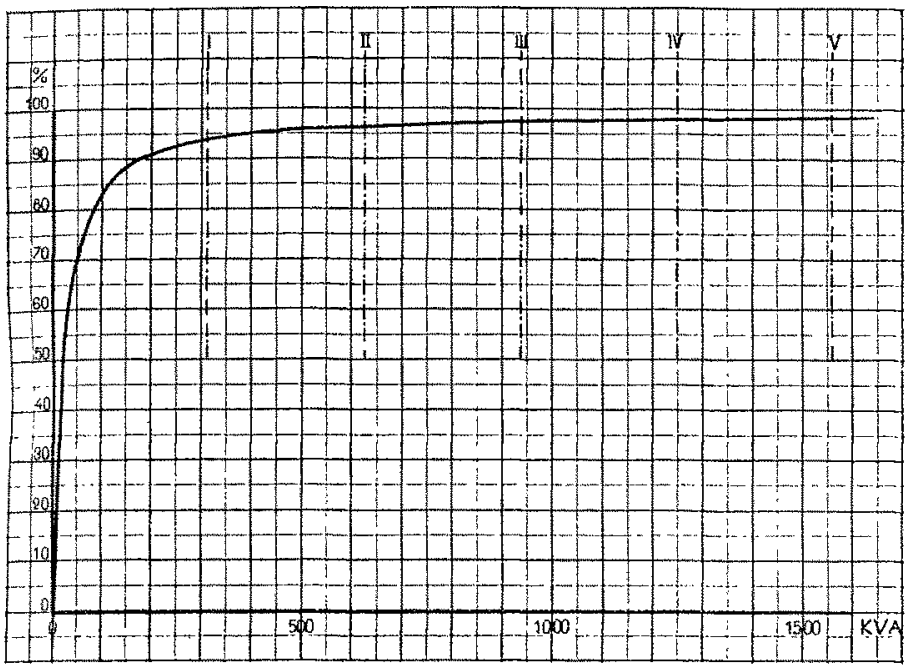

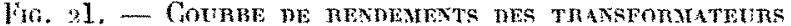

Enfin, l'essai de rigidité des enroulements, réalisé en appliquant pendant 10 minules une tension alternative de 65.000 volts entre les deux enroulement, et entre la huble tension et la masse, n’a permis de polever aurun défaul d'isolement des bobines.

Nous croyons devoir signaler que la sous-slation est munic de toul l'appareillage nécessaire pour le remplissage d'huile des transformateurs, ce qui permet de róluire considérablement la priode de non-ulilisation en cas d'accident. Giost. à nolpe comnaissance, la première fois qu'une sous-stalion est aussi complètement équipéc sous ce rapport.

Chaque transformateur est prolégé par des bobines do solf destines à amoutir les surtensions qui peurent se promduine lors de la mise hors cirenil. Cas bobines d'indurlion sont eonstituécs par un suban de cuive, de dimensions ap' proprieses, enroulé sur un noyau en laiton, do façon ì former une bobine d'umo soixantaine de spires envizon.

le point nentre du primaire et du secondaire de charpo grompe esl mis à la lerre par un parafoudre à corne, de façon à dériver an sol les surlensions qui parviendlaienl aux chrouloments. Ce dispositif implique an isolement parfait de loute l'installation et des lignes, en même lemps qu'il ast une garantie pour la bonne exécution ol la sécuritr de l'insemble.

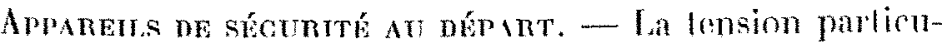
liobrement élevée, l'importance do l'ónergie a transmettre, la longueur considérable de la ligne, el la fréunence des oragor dans la région traversée, exigeaient un systrme do proterliom an départ très efficace el des plus complets. Après unc étude approfondie des mesures ur prendre, on s'arrêta aux dispositifs suivants :

En plus des bobines d'induction montós sur charpue grompe de transformateurs, une bobine identique esl mouléc sur chaque ligne avant sa sorlie de l'usine. Finfon, on a inctalle trois groupes de parafoudres on limileurs de tension ayant chacun in rôle bien déterminé :
$I^{\circ}$ Contre les décharges atmosphériques qui peuvent se produire à la leme, on motre deux mages, on a adoplé des parafoudres ortinaires à cornes, róghín à fo millimòtres, en série avec des résistances liquides.

? Des surélévalions de fousion, igaloment tris dangereuses, peurent se probluire, soit par induction quand une decharge atmosphérique a produil all voikinage des lignes, soit par une charge statique provemant du contact des lignes avec dos muages charges staliquemenl ; des parafoudes à rouleanx, plus sensibles gun los parafoudres à comes, ont fó prevus pour parer à ce langer.

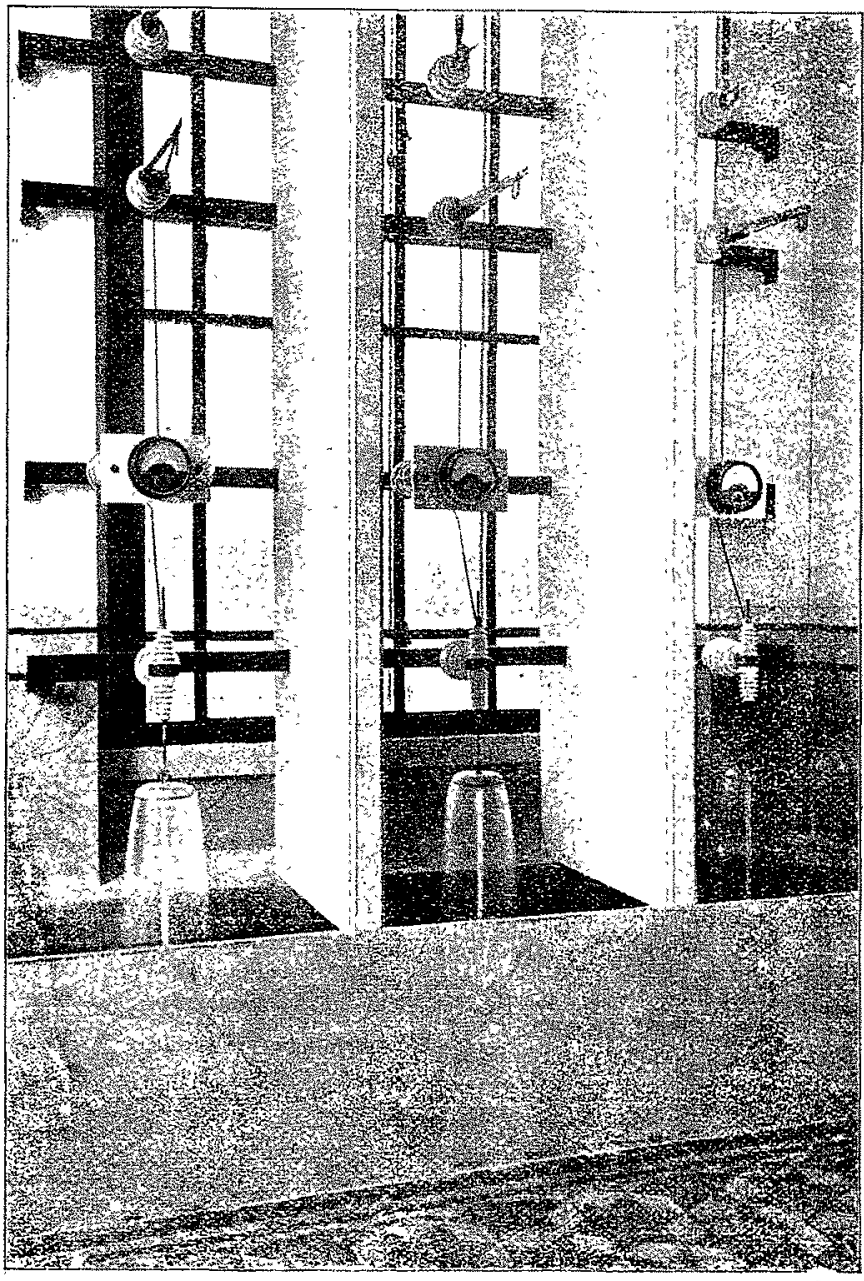

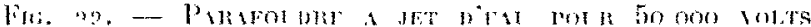

$3^{\circ}$ Enfin, les rantialions do courant on de vollage dommant

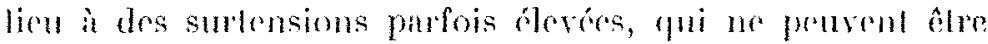
diminées par les deux systomes pródedents, it cause do lenr frequence de de leur pulsalion lres pen différente de la pul-

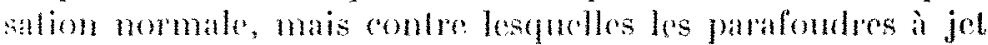

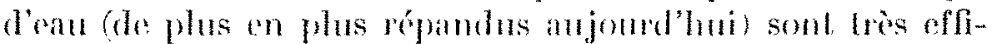

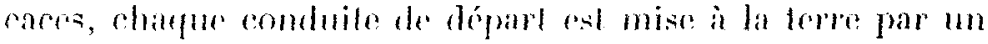
jel d'an, de dimensions of do pression dólomineses, qui

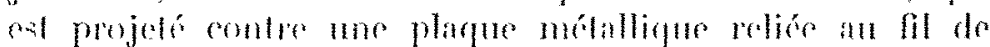

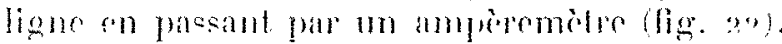

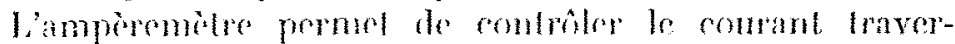
sant linstallation, of set an mone trmps dindieatem de leme, fonte diminution de lintonste dans nne phase indiquant un dofaul disolomont. les jots d'ean ascendants,

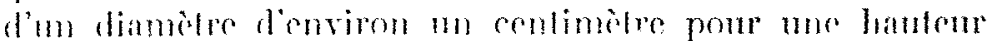

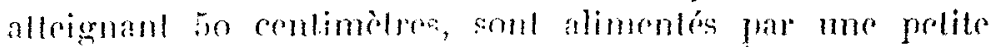

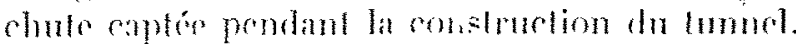

Chayue parafoulde poul chre mis hors circuit an moyen to conteaux. D'autres conteaux permettont do mottro foutos 
les lignes à la terre après leur sortie de la tourelle de départ, de façon à permeltre les réparations en toute sécurité. Enfin, chaque ligne à 50.000 volts est munic d'un interrupteur à huile (non automalique pour éviter le déclenchement subit de tonte la charge).

Une denière précaulion a consisté à relier ontre clles toutes les parlies mélalliques du bàliment non parcourues par le courant, et à les mettre à la tere. La mise à la terre est assurée par trois plaques do for éloignées les unes dos autres et colerrées près du Poschiavino à plusicurs mètres tans la nappe d'eau souteraine. las parafoudre sont reliés aux mêmes plaques. socle on béton, et portenl à leur partie supéricure six supports pour isolateurs à quadruple cloche on porcelaine.

La portée normale est de 120 mètres, mais un certain nombre de portées sont beancoup plus grandes. La plus importanle se trouve à la traverséc du Gravina, près de Colieo. Elle atteint 390 mètres. On a remplacé les câbles ordinaires par des cables formés chacun de ro fils de 2.05 millimòtres de diamètre.

les isolateurs, qui devaient présenter de sérieuses garanlies au point de vue de la solidile mécanique et de lisolement, ont bé foumis par la Suciele 4. R. Ginori, de Pise.

La construction de la ligne, commencée en igo5), a duré

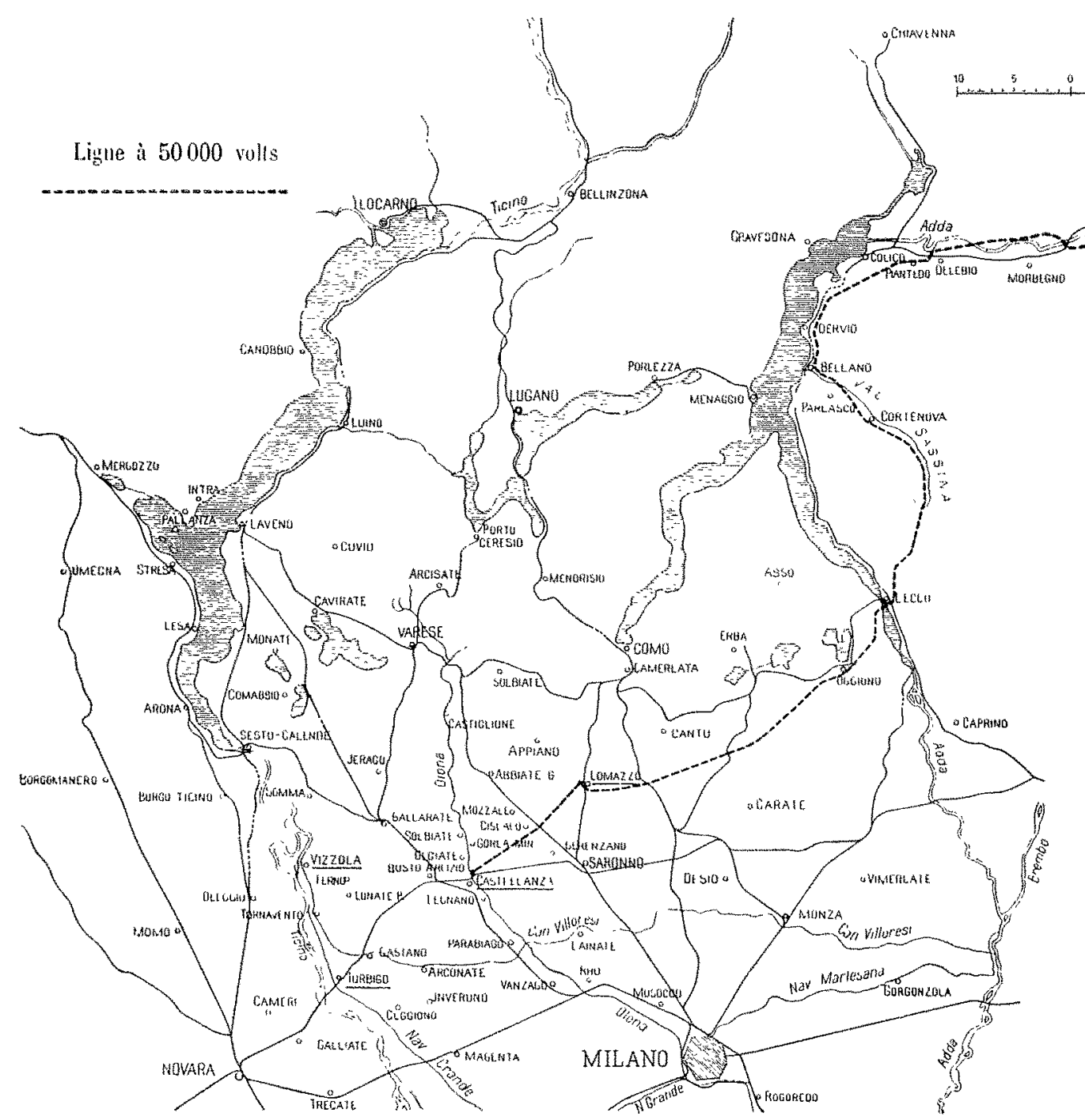

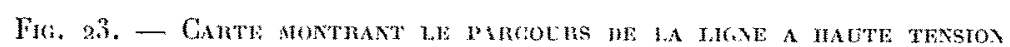

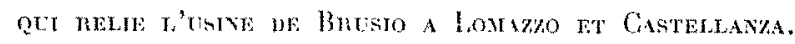

\section{Ligne à haute tension}

La ligne haute tension, qui va de Piallamala à lomazzo ef Castellanza (voir lig. 23), 'n passant par Bellano el lecco śr le lac de Cômo), a été culudéc el exéculéc en entier par la Société Lombarde pous la distribulion de l'́nergie électrique. Elle suit généralement les routes ou les lignes de chemin de fer, ce qui facilite la surveillanee el permet d'éviter les dénivellations brusques. Elle se compose de quatre lignes hiphasées parallòles, montées par doux sur des pylones métalliques en treillis.

Ces pylones, que l'on a construit de quatre types differents pour salisfaire aux diferses contilions locales (angles, traversées de rivière, etc.), ont en moyenne $14^{\mathrm{m}}$ ho de hauteur, et pèsent 700 kilogs. Ils sont cimentés dans un
Une ligne lobphonique, parallele à la ligne a hanle tonsion, rolio les dilfórentes cabines of los points extremes de l'installation. Elle es siture a mo distaner mogenne de vingl mòres de la ligne principale, ef monléc sur poleanx on bois.

\section{Sous-station de Lomazzo}

La station de transformalion de Iomazzo, oit aboulissent les deux lignes venant de Brusio, constilue to véritable centre de distribulion du lransporl d'chergie dont nous venons d'espuisser l'étude, car c'est elle qui fommit le comrant aux différents réseanx de la Sociéló italienne Lombarde.

Celle sous-station a été construite pour le comple te colle Socićlé, d'après lés plans de la Socićté Alioth, qui a également fourni tout l'apparcillage éledrique : Hansformateurs, 
parafouldes, interuptems, etc. Elle est relice par mo ligne friphasce à la sous-station extrême de Castellanza, ce qui permel, grice à un jeu de coutean des plus simples :

${ }^{\circ}$ d'alimenter les deux sous-stations avec les deux lignes darrive en parallele;

? dalimenler chaque sous-slation aved une ligne, ou are les drux lignes montécs en parallèle ;

$3^{\circ}$ d'alimenter Lomazzo par Castellanza, où so frouvent dis groupes a vapeur de secours, en cas daccillent sur les deu ligues principales.

Vous ne nous altarderons pas à décrire la slation de lomazon qui a été conslruito d'après les mêmes idóes générales fue celle de Piallamala. Conlentons-nous de signaler qu'elle conlicul six transformaleurs monophasés de $1250 \mathrm{~K}$. V. A.: abaistant la teusion à $r$ ooo rolts (voltage nomal des réseans de la société Lombarde), et six lmanformateurs friphases do 5oo kilowatls, de y noo ${ }^{\prime} 20$ ooo volls, deslines au transpont de 3 oon kilowalls à Come. Les apparpils de sécuritr de mise à la terre à l'entrée et à la sortie des lignes sont lo mèmes qu'à Plattamala el nous n'y reviendrons pits.

l.es transformateurs, toutefois, sont à renlilation forcé, ft chla paree que lon voulait voir, pendant la période d'essais, fuel serait te meilleur systène des deux. On a pu conslalcr par la suite que lous les denx remplissaient parfaitemurut leur ròle. On peut cependant préférer le transformateur à rentilation force comme dant d'unc surveillance el d'un colretien plus faciles. Par contre, lo rendement est. liggerement dimimú, et un arrel accidentel, loujours possible du ventilateur, est dangereux, car loul disposilif de refroidissement est alors supprimé. On a d'ailleurs prévu fe eas ì Lomazzo, en installant deux puissants ventilateurs font lim est toujours en réserye.

\section{Sous-station de Castellanza}

La sous-stalion de Castellanza, point extrême de la ligne, est une anmexe de la centrale à vapem que possède la Sociélé lombarde, el qui comprend deux turbomallernaleurs do 5.000 chevaux, el deux groupes à vapeur de 3.500 chevaux. Cetle usine sert de réserve aux centrales de Turbigo el Vizzola dont nous avons dil un mol au dóbul. II ćtail done nalurel de la relier an résenu général.

Nous atons dit plus haul comment il élail possible d'effectuer culre Lomazzo of Castellanza, Iontes les combinaisons possibles permeltant lalimentalion te Tune on laulre des sous-slations, voire de toutes les dous de différentes foçons.

La disposition inkérieme de la sous-station, eonçue d'ajues des plans analognes a coux des slations do Piallamala de lomazzo, no présente aucune particularita, si co n'ost que la place faisant déful, on ful oblige de disposer les conthiles ol apparoils divers dans trois chages differents.

Ene modification mérite cependant d'ulre signalé ici : les relais des disjoncleurs aubomaligues penvent elro branchés sur la bobine do díchenement de linterumplen, de faron que ce soil cenx du circull hate lension qui déclenphent fuand Gatclanza fonclionne comme sous-station, ef coux da circuil basse lemsion quand elle fometionne comme station génćntrice, cola en tertn do son ròle crenlue eomme usine de secoms. Gest égaloment pour la mene rison que dos bobines dimoluction ont dé placés sur les leux enroulements des transformatours.

\section{Mise en marche}

Dès lo mois de novembre 1906 , les installations étaient suffisamment avancées pour que l'on pût songer à une mise en exploitation graduelle et, des le mois suivant, on pût procéder aux essais de mise en charge. Le 7 decembre, on fit les premiers essais à 10.000 volts entre les sous-stations de lomazo of Castellanza, puis sur toule la liene, depnis Pinthamala, ot après des rssais de débit en charge sur résislances. Ie 8 mars $190 \%$, l'explotitation régulièc commenga avec whe charge do 3.000 bilowalts. Huit mois apres, elle stail passé à ra.0oo hilowalls el dans le courant de l'amnée rgos, ello alleignit rapidement 16.000 kilowalls, chiffer prévu an conlrat passé cutre les dens sociótés intóressées.

les prévisions des ingéniemrs ef des constructeurs chargés de l'entreprise avaicnl ainsi éle réalisées, ol an-dela, sans aucun incident, el cest là mo paison de plus gui fait que co transport de puissance de aso kilometres, dont nous "latons pu donner, ici, qu'me très sommaire el bicn imparfaite description, fransport remarquable pat son agencement irréprochable et les dispositions parliculièrement heureuses aloptés, peut être considéró, à jusle litre, commo l'un dos phus parfals existant actuellement en Furope.

Georges Ferroux,

Chargi de Combinones

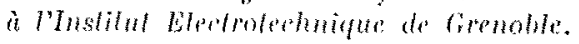

\section{IIYDR.ULLQUE}

\section{LA DURANeE ET SON UTILISATION}

(Sulle)

\section{Nécessité de régulariser le régime de la Durance}

les indications que nous avons données an sujet du débit de la Durance montrenl combien est variable le régime de celle riviere lomenticlle. A Rolssel, alle descend it $19 \mathrm{nt}^{3}$ par les basses onx dhixr, pont alleindere en temps de crue 1.5oo el même r.800 $\mathrm{m}^{3}$ par seconde. A Mimbean, sos débits refremes on déte th el do 6.000 medes cubes.

Ce regime torrentiel sicommole nal ares les besoins do l'agriculture et de Tindustrie. De plus, les grandes crues sont tres dommageables aux riverins don elles corrodent les terres et los inomlent quelquefois. C'ost surtout dans la basse Provence, en aval de Mirabeau, que les inondations ont elo désastrouses. On a conserví, nolamment, le souvenir des erues de $1843,1856,1883$ ol 1886 , qui ont emporte des ponti, submorgé el pompu des digues of inomde de vastes plaines cullivées.

Pónulips d'ent agricoles. - Pendant Imagemps, le dóbit de la Durance a kf toujours suflesm pour astrer largement latimentalion des canans dirrigation qui on sont dépi-

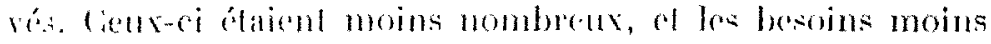

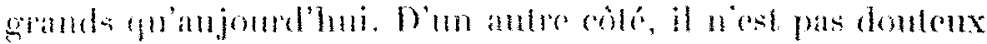

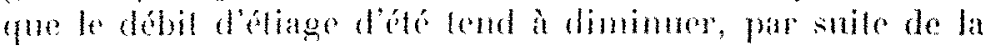

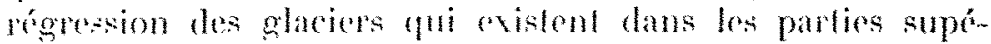

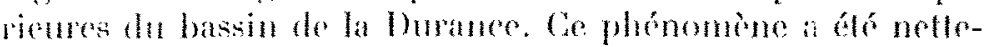

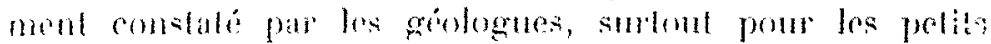

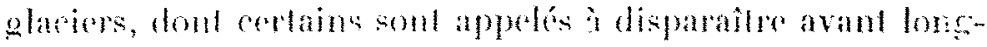
louly $x$

Ouoi quil on soll, il est me fat certain, cost que, depuis 18,5, pour to pas remonter phus hat, i] arive on moyenne une amnce sur deus que to dihit disponible est insuffisan 